\title{
The relationship between androgen levels and human spatial abilities
}

\author{
VALERIE J. SHUTE, JAMES W. PELLEGRINO, LAWRENCE HUBERT, \\ and ROBERT W. REYNOLDS \\ University of California, Santa Barbara, California
}

\begin{abstract}
Individual differences on spatial tasks were examined relative to differences in free-androgen levels. A spatial test battery was administered to 91 males and females who differed in freeandrogen levels as determined by a radioimmunoassay. Polynomial regression analyses yielded significant curvilinear functions relating spatial scores and androgen levels. A second study, conducted with a group of 33 males and females, replicated the basic findings of the first experiment.
\end{abstract}

Psychologists and educators concerned with individual differences in cognitive abilities have been particularly interested in spatial abilities, since male superiority on tasks requiring these abilities is among the most consistent findings in the abilities literature (Maccoby \& Jacklin, 1974). Tests requiring spatial-visualization and/or spatial-orientation decisions yield reliable sex differences for adolescent and adult populations but not for younger populations (Maccoby \& Jacklin, 1974; McGee, 1979).

Since sex differences in spatial skills begin to appear at about the age of 8 years, it has been hypothesized that they are partially related to differences in gonadal hormone production. Between the ages of 8 and 10 years, males begin producing increased levels of testosterone, with the levels reaching asymptote during adolescence (Gandy \& Peterson, 1968). Coincident with this is the finding that, with subjects 13 years of age or older, males generally perform consistently better than do females on tests of spatial ability (Maccoby \& Jacklin, 1974). This sex-related difference in spatial abilities continues throughout adulthood, with a general decline in ability for both sexes paralleling a gradual decrease in hormonal levels over adulthood (Wittig \& Petersen, 1979).

Since secondary sex characteristics are under the influence of gonadal hormones, there have been attempts to study their relationship to scores on spatial tests. Petersen (1976) investigated the relationship between somatic measures of hormone influence and cognitive performance in both males and females. At ages 16 and 18 years, androgynous males (i.e., less stereotypic in physical appearance) were better at spatial than verbal tasks, whereas males with more extremely masculine body types were better at verbal than at spatial tasks.

James W. Pellegrino's mailing address is: Department of Education, University of California, Santa Barbara, California 93106.
For females, those more androgynous in appearance (e.g., small breasts, wide shoulders, narrow hips, and solid muscles) were better than more "feminine" counterparts on spatial tasks. Thus, androgynous males and females tended to excel at spatial tasks in comparison with those who were more sex stereotypic in appearance.

The Petersen (1976) study implies that a curvilinear (quadratic) relationship may exist between androgen levels and performance on spatial tasks. Individuals with extremely high or low levels of androgen would be expected to show poorer spatial performance. Studies of individuals with biological abnormalities affecting androgen levels are consistent with such a hypothesis. Examples include males who demonstrate the androgen insensitivity syndrome, females with Turner's syndrome, and males with severe kwashiorkor (Alexander \& Money, 1966; Dawson, 1972; McGee, 1979).

Hier and Crowley (1982) investigated androgens and spatial abilities in a group of adult male patients whose sexual maturity had been delayed because of unexplained low levels of male hormones (androgens). These men performed significantly less well on standard tests of spatial ability than did healthy male volunteers. In addition, this group performed worse on the tests than did a small group of men who developed androgen deficiency after puberty. Hier and Crowley concluded that high androgen levels need to be present at puberty for normal development of spatial abilities.

The data that are available on the relationship between androgen levels and spatial ability are limited in two important ways. First, previous methods for assessing androgen levels in normal individuals have been very imprecise, relying more on physical than on direct physiological indexes. Second, the cases of extremely low levels of androgen represent anomalous groups rather than biologically normal males and females, and thus represent the extremes of the normal population range. The purpose of the present study was to examine 
more precisely the form and level of the relationship between androgen levels and spatial performance in a sample of normal males and females. Spatial performance was assessed by standardized spatial-ability tests, and androgen levels were assessed by radioimmunoassay (RIA) of free testosterone in blood samples.

Free testosterone was used as a hormonal index in this study because it can pass the blood-brain barrier (unlike bound testosterone) and thus exert its influence on various sites in the brain that are associated with cognitive processing. An example is hippocampal pyramidal cells that have been implicated by lesion and electrophysiological studies as being important in spatial behavior. This site, as well as the visual cortex, shows evidence of sexual differentiation. Furthermore, testosterone and estradiol have been shown to have opposite effects on physiological responses of hippocampal CAI pyramidal cells in male and female rats (McGlone, 1980). In addition to the localized effects of testosterone on specific brain sites, there may be a general stimulating effect of testosterone. Previous work has suggested that testosterone tends to lower monoamine oxidase (MAO) activity in humans and thus results in an increase in catecholamine levels in the activating sympathetic nervous system (Broverman, Klaiber, Kobayashi, \& Vogel, 1968).

\section{EXPERIMENT 1}

\section{Method}

Subjects. The 91 subjects represented undergraduate and graduate students at the University of California at Santa Barbara. The age range was 16 to 41 years, with a mean age of 24.5 years. Nine of the subjects were older than 31 years, and only one subject was 41 years old. As noted earlier, spatial abilities reach a plateau (for both sexes) at puberty and begin declining in the late 20 s. The "aging" effect (i.e., the decline in spatial performance) was investigated, but because of the relatively restricted age range of the subjects, the aging effect proved not to be an important factor. All analyses were done for the entire sample and also with those subjects older than 31 years omitted. The results showed no changes in fit and virtually identical coefficients for the least squares regression polynomials.

Procedure. The subjects were tested in small groups, and all testing was done at the same time of day (11:00 a.m.). The control of testing time was done to remove any confounding associated with diurnal fluctuation in androgen levels. After an initial questionnaire was completed, blood samples were obtained from each subject by pricking the finger tip with a lancet. Using an RIA technique, the blood samples were separated and the plasma was frozen for subsequent analysis. The subjects were then administered a battery of six timed spatial-ability tests. The tests were selected from the French Reference Kit for Cognitive Factors (French, Ekstrom, \& Price, 1969) and included two tests from each of three factors: flexibility of closure (Cf), spatial orientation (S), and visualization (Vz).

The RIA was based on a procedure that compared the ratio of bound to unbound radioactive testosterone, that is, testosterone labeled with tritium at six sites. Following ether extraction of unlabeled free testosterone from blood plasma, the unlabeled and radioactive testosterone were incubated together with an antibody that is specific for binding testosterone. The ratio of bound to unbound radioactive testosterone varies as a function of the amount of unlabeled free testosterone in the sample. This ratio variation results from competition between the unlabeled and radioactive testosterone for binding sites. The ratio of bound to unbound radioactive testosterone is compared with a standard curve determined prior to the experiment. This curve serves as the basis for estimating the amount of free testosterone in a given plasma sample. In this study, a reliability check was done on the estimation procedure (the females' plasma samples were used because the expected range of values is much more restricted). This analysis yielded a reliability of .75 (correlation coefficient).

\section{Results}

Four spatial-ability scores were derived for each subject: an overall spatial score based on the average of all tests and a score for each pair of tests representing a major factor. The results of a comparison between males and females are shown in Table 1 . In all cases, significant mean differences were obtained. The $t$ values $(\mathrm{df}=89)$ were $3.23,2.04,2.99$, and 2.81 for the overall mean, $\mathrm{Cf}, \mathrm{S}$, and $\mathrm{Vz}$ scores, respectively. The respective $\mathrm{p}$ values were $.002, .04, .004$, and .006 . Thus, the data are consistent with the existence of an overall sex difference in spatial ability in adult samples. Furthermore, the differences are greatest for the more central spatialaptitude factors, namely spatial orientation (S) and spatial visualization $(\mathrm{Vz})$.

The RIA of testosterone levels produced a single score for each subject. As expected, there was a significant sex difference in testosterone levels, with a mean of $11.2 \mathrm{ng} / \mathrm{ml}$ (nanograms per milliliter) for males versus a mean of $2.3 \mathrm{ng} / \mathrm{ml}$ for females [ $\mathrm{t}(89)=13.08, \mathrm{p}<$ $.001]$. These obtained values are higher than values previously reported in the literature on normal freetestosterone levels for males and females. The crossreactivity of the antibody was presumably binding with other androgens that are biochemically similar to testosterone. Therefore, we will be discussing the relationship between androgens, in general, and spatial skills.

The relationship between androgen levels and spatial ability was evaluated by polynomial regression analyses. Separate analyses were done for the overall spatial mean and the means for each separate spatial factor. In addition, analyses using the actual values from the RIA and log-transformed values were conducted. The results of all of these analyses were very similar, and thus the only analysis reported is that for the relationship between the overall spatial mean and the log transformed andro-

Table 1

Spatial Aptitude Measures as a Function of Sex

\begin{tabular}{lccccc}
\hline & \multicolumn{2}{c}{ Males } & & \multicolumn{2}{c}{ Females } \\
\cline { 2 - 3 } \cline { 6 - 6 } Aptitude Measures & Mean & SD & & Mean & SD \\
\hline Cf-Factor & 51.9 & 14.9 & & 45.7 & 14.2 \\
S-Factor & 69.5 & 17.4 & & 58.8 & 16.5 \\
Vz-Factor & 53.9 & 18.0 & & 43.3 & 18.0 \\
Overall Spatial Mean & 58.6 & 13.1 & & 49.4 & 13.9 \\
\hline
\end{tabular}

Note-Males, $N=43$; Females, $N=48$. 
gen values. The least squares method was used to determine best-fitting curves, with testing done for the range of first-order to fifth-order polynomials. The analyses indicated that the best fit was obtained by the thirdorder polynomial, with fourth- and fifth-order components failing to contribute additional significant variance. Figure 1 shows the scatterplot of the raw data and the curve representing the best-fitting function. The multiple $r$ for the function shown in Figure 1 was $.39(\mathrm{p}<.05)$. Analysis of the separate trend coefficients indicated that only the quadratic and cubic trends were significant.

The data shown in Figure 1 illustrate the overall difference between males and females in androgen levels and the difference in the range of values obtained within each sex. The general relationship between androgen levels and spatial ability seems to be a function of separate trends within the male and female groups. To investigate this more precisely, separate polynomial regression analyses were conducted for each sex. The polynomial regression analysis of the male data yielded a highly significant third-order polynomial with a multiple $\mathrm{r}=.59(\mathrm{p}<.001)$. Analysis of the linear, quadratic, and cubic trends showed that all three trend components were significant. The right panel of Figure 2 shows the male data and the best-fitting function.

Similar polynomial regression analyses of the female data indicated that there was a positive linear trend that did not reach statistical significance $(r=.12, p>.05)$. The female data, together with the best-fitting linear function, are shown in the left panel of Figure 2. The female data were not a function of birth control pill usage (which influences testosterone levels), since those individuals taking the pill were evenly distributed throughout the graph. However, the menstrual phase does result in variability in normal women's testosterone levels, and in this study, control of the menstrual phase (follicular vs. luteal) could not be done. It was also not possible to determine the test-retest reliability of the androgen

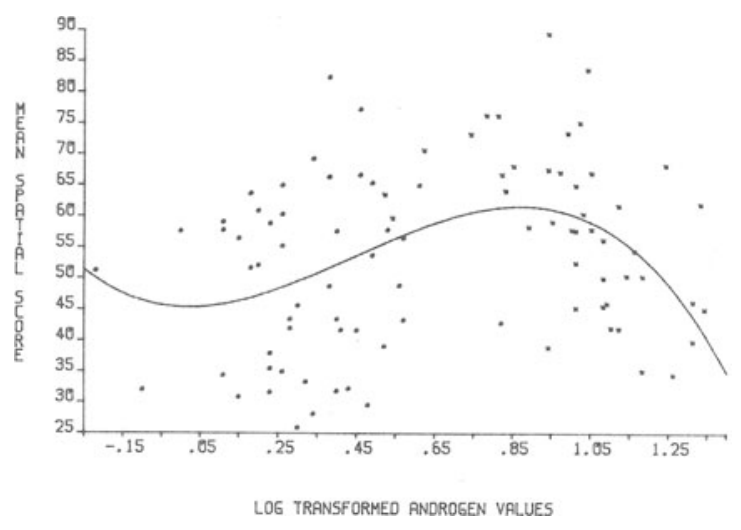

Figure 1. Scatterplot of the data representing the relationship between androgen levels and spatial test score means together with the best-fitting third-order polynomial.
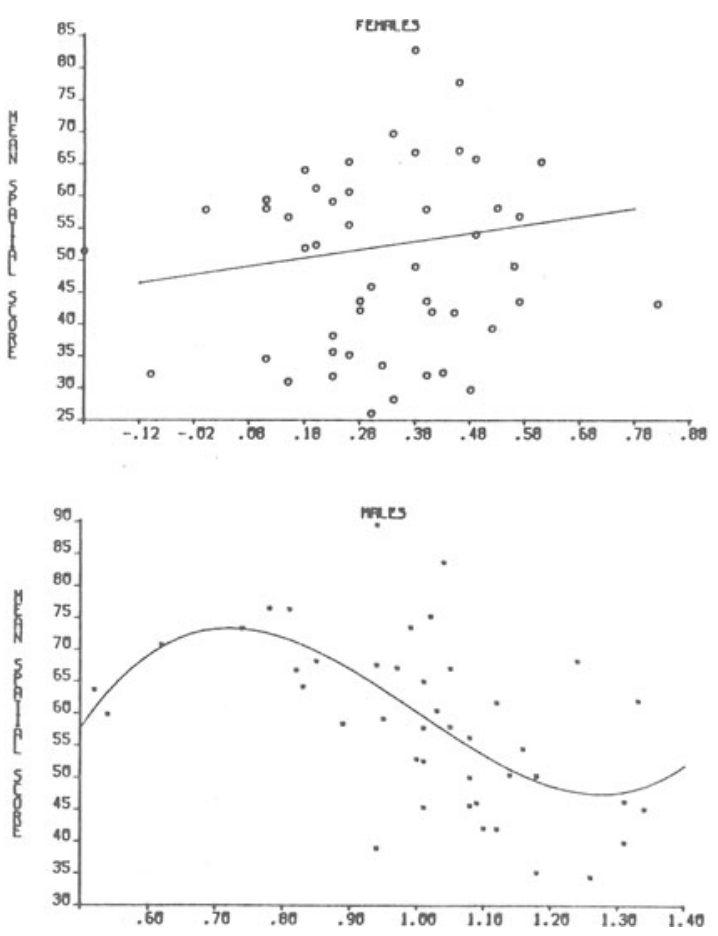

Figure 2. Separate scatterplots of the female and male data, with the best-fitting functions for each sex.

levels for the females. Given that free-testosterone levels are restricted in females and are subject to menstrual fluctuation, it is entirely possible that the failure to find a significant linear relationship between testosterone and spatial scores is due to the low reliability of the former when estimated from a single sample.

The separate analyses of each sex support the general interpretation that the overall curvilinear trend observed for the entire sample is a function of a curvilinear trend for males and two opposite linear trends for males and females, the latter of which is nonsignificant when evaluated separately. Although the restriction in range of testosterone levels in the female sample presents interpretative difficulties, it was found that the females had a positive (albeit statistically nonsignificant) trend consistent with the trend components observed for males.

\section{EXPERIMENT 2}

Experiment 1 provided support for the existence of sex differences in spatial ability that are partially related to differences in gonadal hormone levels. The purpose of Experiment 2 was to provide a further test of this relationship with a new sample of individuals. It was hypothesized that males with high androgen levels (relative to other males) would score lower on spatialability tests than would males with low androgen levels. Conversely, females with high androgen levels (relative to other females) would score higher on spatial-ability 


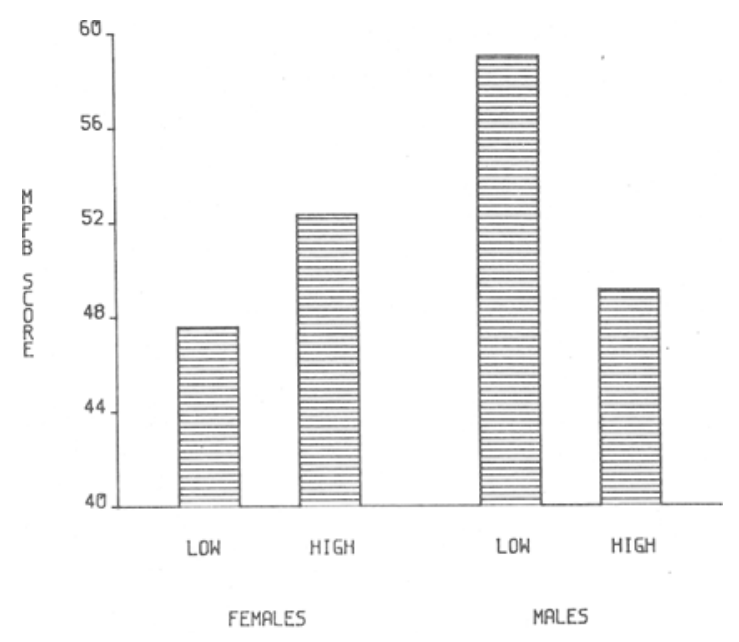

Figure 3. Mean MPFB scores as a function of sex and androgen level within sex.

tests than would females with low androgen levels. To test for such an interaction, a contrastive-groups approach was used, with androgen level (high vs. low) defined separately for each sex.

\section{Method}

Subjects. The subjects in this study represented undergraduate and graduate students at the University of California at Santa Barbara. Eighteen females and 15 males were initially tested. From these 33 subjects, 24 who represented the six highest and lowest androgen values within each sex were selected.

Procedure. Three spatial-ability tests were administered to the subjects individually and in small groups. The spatial tests used were the Primary Mental Abilities Test (PMA) (Thurstone, 1965), the Minnesota Paper Form Board Test (MPFB) (Likert \& Quasha, 1970), and the Comprehensive Ability Battery Space Test (CAB-S) (Hakstian \& Cattell, 1976); these tests provide a range of spatial-processing tasks, from relatively simple two-dimensional mental rotation (PMA, CAB-S) to more complex spatial visualization (MPFB). Following the test battery, blood samples were collected for subsequent analysis by an RIA.

\section{Results}

Two dependent measures were analyzed in separate analyses of variance (ANOVAs): the MPFB score and the mean for the two mental-rotation tests (PMA and CAB-S). The variables in the ANOVA were sex and androgen level (high vs. low). A significant sex $\mathrm{x}$ androgen level interaction was obtained in the analysis of MPFB scores $[F(1,20)=4.32, p=.05]$. This interaction is shown in Figure 3. No other effects were significant. The analysis of scores on the mental-rotation tests failed to indicate any significant main effects or interaction, although the latter was in the predicted direction.

\section{GENERAL DISCUSSION}

Both experiments provided support for the existence of a curvilinear relationship between androgen levels and spatial abilities, with maximal spatial performance occurring for lowandrogen males and for high-androgen females. When females and males are considered together, a significant curvilinear relationship was obtained between a physiological index representing androgen level and scores on standard spatial tests. This overall relationship appears to be the result of different trends within each separate sex group. The conclusion that spatial ability is related to androgen levels is not an artifact of a confounding between sex, androgen levels, and sex differences on spatial tests. The male data indicate a very robust relationship between hormonal levels and spatial performance.

Finally, it is important to note that no single factor can be assumed to underlie sex and individual differences in complex spatial ability. Theories of sex and individual differences must acknowledge the contributory influences of environmental, cultural, genetic, and many other factors.

\section{REFERENCES}

Alexander, D., \& Money, J. Turner's syndrome and Gerstmann's syndrome: Neurophysiologic comparisons. Neurophysiologia, 1966, 4, 265-273.

Anderson, J. R. Cognitive Psychology. New York: Academic Press, 1975.

Broverman, D. M., Klaiber, E. L., Kobayashi, Y., \& Vogel, W. Roles of activation and inhibition in sex differences in cognitive abilities. Psychological Review, 1968, 75, 23-50.

Dawson, J. L. M. Effects of sex hormones on cognitive style in rats and men. Behavior Genetics, 1972, 2, 21-42.

French, J. W., Ekstrom, R. B., \& Price, L. A. Manual for Kit of Reference Tests for Cognitive Factors. Princeton, N.J: Educational Testing Service, 1969.

Gandy, H. M., \& Peterson, R. E. Measurement of testosterone and 17-ketosteroids in plasma by the double isotope dilution derivative technique. Journal of Clinical Endocrinology and Metabolism, 1968, 28, 949-977.

Hakstian, A. R., \& CATtell, R. B. Comprehensive ability battery. Champaign, Ill: Institute for Personality and Ability Testing, 1976.

Hier, D. B., \& Crowley, W., Jr. Spatial ability in androgendeficient men. New England Journal of Medicine, 1982, 306, 1202-1205.

Likert, R., \& Quasha, W. H. Revised Minnesota Paper Form Board Test. New York: Psychological Corporation, 1970.

Maccoby, E. E., \& JACKLin, C. N. The psychology of sex differences. Stanford, Calif: Stanford University Press, 1974.

McGee, M. G. Human spatial abilities: Sources of sex differences. New York: Praeger, 1979.

McGlone, J. Sex differences in human brain asymmetry: A critical survey. Behavioral and Brain Sciences, 1980, 3, 215-263.

Petersen, A. C. Physical androgyny and cognitive functioning in adolescence. Developmental Psychology, 1976, 12, 524-533.

Thurstone, T. G. Primary mental abilities. Chicago: Science Research Associates, 1965.

Wittig, M. A., \& Petersen, A. C. (Eds.). Sex-related differences in cognitive functioning. New York: Academic Press, 1979.

(Manuscript received for publication July 18, 1983.) 